\title{
Regional - National - Supranational. Wahlkampfberichterstattung in Deutschland auf verschiedenen Ebenen des politischen Systems*
}

\author{
Jürgen Wilke und Melanie Leidecker
}

Wahlen und Wahlkämpfe sind in demokratischen Systemen entscheidende Weichenstellungen des politischen und gesellschaftlichen Lebens. Daher hat ihre Erforschung inzwischen eine lange Tradition. Dies gilt nicht nur für die Politikwissenschaft, sondern auch für die Publizistik- und Kommunikationswissenschaft. Seit der klassischen Erie County-Studie $1940^{1}$ ist immer wieder untersucht worden, welche Rolle die Medien der Massenkommunikation bei Wahlen von Parlamenten oder Präsidenten spielen. Diese Studien dokumentieren auch, wie sich im Laufe der Jahrzehnte die Wahlen und Wahlkampagnen, die Medien und ihre Berichterstattung sowie das Wahlverhalten der Bürger verändert haben ${ }^{2}$. Dabei beschäftigte man sich zumeist mit Wahlen auf der nationalen politischen Ebene. Doch sind die Bürger in Demokratien häufig auch noch zu anderen Wahlen aufgerufen.

Ein Paradebeispiel dafür sind die seit 1979 in den Mitgliedsländern der Europäischen Union (EU) veranstalteten Wahlen zum Europäischen Parlament (EP), die inzwischen regelmäßig untersucht werden ${ }^{3}$. In einigen Fällen wurden auch schon Vergleiche der Berichterstattung über nationale und EP-Wahlen angestellt ${ }^{4}$.

* Die Durchführung der Untersuchung wurde durch finanzielle Unterstützung der FAZIT-Stiftung ermöglicht, wofür wir an dieser Stelle danken möchten.

1 Vgl. Paul F. Lazarsfeld / Bernard Berelson / Hazel Gaudet, The People's Choice. How the Voter Makes up his Mind in a Presidential Campaign, New York 1944.

2 Vgl. Bernd-Peter Lange / David Ward (Hrsg.), The Media and Elections. A Handbook and Comparative Study. Mahwah 2004; Jesper Strömbäck / Lynda Lee Kaid (Hrsg.), The Handbook of Election News Coverage around the World, New York 2008.

3 Vgl. Jay G. Blumler (Hrsg.), Communication to Voters. Television in the First European Parliamentary Elections, London 1983; Christina Holtz-Bacha (Hrsg.), Europawahl 2004. Die Massenmedien im Europawahlkampf, Wiesbaden 2005; Claes H. de Vreese / Edmund Lauf I Jochen Peter, The Media and European Parliament Elections: Second-rate Coverage of a Second-order Event?, in: Wouter van der Brug / Cees van der Eijk (Hrsg.), European Elections and Domestic Politics. Lessons from the Past and Scenarios for the Future, Notre Dame 2007, S. 129 - 147; Michaela Maier / Jesper Strömbäck / Lynda Lee Kaid (Hrsg.), Political Communication in European Parliamentary Elections, Aldershot 2011; Jens Tenscher (Hrsg.), Superwahljahr 2009. Vergleichende Analysen aus Anlass der Wahlen zum Deutschen Bundestag und zum Europäischen Parlament, Wiesbaden 2011.

4 Vgl. Jürgen Wilke / Carsten Reinemann, Invisible second-order campaigns? A Longitudinal Study of the Coverage of the European Parliamentary Elections 1979 - 2004 in Four German Quality Newspapers, in: Communications, 32. Jg. (2007), H. 3, S. 299 - 322; Hajo G. Boomgarden / Claes H. de Vreese / Holli A. Semetko, „Hast es nicht gesehen?!“ Haupt- und Nebenwahlkämpfe in deutschen Fernsehnachrichten, in: Jens Tenscher (Hrsg.), a.a.O. (Fn. 3), S. 181 - 197; Jesper Strömbäck / Lars W. Nord, Still a Second Order Election: Comparing Swedish Media Coverage of the 2004 European Parliamentary Election and the 2002 National Election, in: Lynda Lee Kaid (Hrsg.), The EU Expansion. Communicating Shared Sovereignty in the Parliamentary Elections, New York u.a. 2008, S. 137 - 152; Jürgen Wilke / Christian Schäfer / Melanie Leidecker, Mit kleinen Schritten aus dem Schatten. Haupt- und Nebenwahlkämpfe in Tageszeitungen am Beispiel der Bundestags- und Europawahlen 1979 - 2009, in: Jens Tenscher (Hrsg.), a.a.O. (Fn. 3), S. 155 -179 . 
Bisher weitgehend ausgeblendet blieb jedoch, dass im lokalen oder regionalen Raum ebenfalls Wahlen stattfinden, in der Bundesrepublik Deutschland also auf der regionalen (Landtage) und kommunalen (Stadt- und Gemeinderäte) Ebene. Auch diesbezüglich haben sich die Politikwissenschaft und die Publizistikwissenschaft ähnlich verhalten. Zwar liegen seit den 1970er Jahren einzelne politikwissenschaftliche Fallstudien zu verschiedenen Landtagswahlen vor. Während Roth und Seibt ${ }^{5}$ die Rolle der Parteien bei der Bremer Bürgerschaftswahl 1976 untersuchten, ging es Sarcinelli ${ }^{6}$ und seinen Mit-Autoren bei der Landtagswahl von Rheinland-Pfalz 1983 um eine „holistische“ Betrachtung von Parteiprogrammatik, Planung und Verlauf des Wahlkampfs sowie von Wahlergebnis und Wählerverhalten. Ähnliche Bestandsaufnahmen mit diversen zusätzlichen Fragen wurden erst wieder zu den Landtagswahlen in Baden Württemberg $2001^{7}$ sowie in Rheinland-Pfalz und Baden-Württemberg 2006 vorgelegt ${ }^{8}$. Weitere Einzelstudien finden sich in der ZParl. Zuletzt widmete die Zeitschrift anlässlich des „Superwahljahrs“ 2011 fast ein ganzes Heft den Landtagswahlen in fünf Bundesländern? .

In der Politikwissenschaft hatte man sich im Übrigen vor allem dem Verhältnis von Landtagswahlen und Bundestagswahlen zugewandt, einer Frage, die Dinkel ${ }^{10} 1977$ als einer der ersten aufgegriffen hatte. Ihm folgten vereinzelte Folgestudien ${ }^{11}$ bis hin zu einer vor wenigen Jahren publizierten kollektiven Analyse des Wählerverhaltens bei Landtagswahlen in Deutschland ${ }^{12}$. Eine neue Studie dazu wurde ebenfalls kürzlich in der ZParl vorgelegt ${ }^{13}$. Die Medien der Massenkommunikation kamen in solchen politikwissenschaftlichen Untersuchungen aber so gut wie nicht vor oder wurden allenfalls als Informations- und Zitatquelle benutzt beziehungsweise kursorisch behandelt.

Merkwürdigerweise waren es gleich zwei Landtagswahlen im Jahr 2000, die erstmals Gegenstand mehrerer kommunikationswissenschaftlicher Studien wurden. Die nordrhein-

5 Vgl. Reinhold Roth / Peter Seibt (Hrsg.), Etablierte Parteien im Wahlkampf. Studien zur Bremer Bürgerschaftswahl 1976, Meisenheim am Glan 1979.

6 Vgl. Ulrich Sarcinelli (Hrsg.), Wahlen und Wahlkampf in Rheinland-Pfalz. Beiträge für die politische Bildungsarbeit aus Anlaß der Landtags- und Bundestagswahlen am 6. März 1983, Opladen 1984.

7 Vgl. Josef Schmid / Honza Griese (Hrsg.), Wahlkampf in Baden-Württemberg. Organisationsformen, Strategien und Ergebnisse der Landtagswahl vom 25. März 2001, Opladen 2002.

8 Vgl. Josef Schmid / Udo Zolleis (Hrsg.), Wahlkampf im Südwesten. Parteien, Kampagnen und Landtagswahlen 2006 in Baden-Württemberg und Rheinland-Pfalz, Münster / Hamburg 2007.

9 Vgl. unter anderem Heiko Gothe, Die rheinland-pfälzische Landtagswahl vom 27. März 2011: Dosierter Machtwechsel in Mainz, in: ZParl, 42. Jg. (2011), H. 4, S. 764 - 783; Oscar W. Gabriel / Bernhard Kornelius, Die baden-württembergische Landtagswahl vom 27. März 2011: Zäsur und Zeitenwende?, in: ZParl, 42. Jg (2011), H. 4, S. 784 - 804.

10 Vgl. Reiner Dinkel, Der Zusammenhang zwischen Bundes- und Landtagswahl-Ergebnissen, in: PVS, 18. Jg. (1977), H. 2/3, S. $348-359$.

11 Vgl. Georg Fabritius, Wechselwirkungen zwischen Landtagswahlen und Bundespolitik, Meisenheim 1978; Frank Decker / Julia von Blumenthal, Die bundespolitische Durchdringung der Landtagswahlen. Eine empirische Analyse von 1970 bis 2001, in: ZParl, 33. Jg. (2002), H. 1, S. 144 - 165; Daniel Hough / Charlie Jeffery, Landtagswahlen: Bundestestwahlen oder Regionalwahlen?, in: ZParl, 34. Jg. (2003), H. 1, S. $79-94$.

12 Vgl. Kerstin Völkl / Kai-Uwe Schnapp / Everhard Holtmann / Oscar W. Gabriel (Hrsg.), Wähler und Landtagswahlen in der Bundesrepublik Deutschland, Baden-Baden 2008.

13 Vgl. Christopher Baethge, Rolle im Bund und Erfolg im Land - eine parteienbezogene Analyse der Landtagswahlen von 1949 bis 2010, in: ZParl, 42. Jg. (2011), H. 4, S. 568 - 586. 
westfälische wurde vor allem hinsichtlich der „Amerikanisierung“, des Agenda-Setting und Agenda-Building sowie der Medialisierung untersucht ${ }^{14}$. Außerdem wurde der Medialisierung sowie der Veränderbarkeit von Kandidatenimages im Landtagswahlkampf durch Medien nachgegangen ${ }^{15}$. Bei der schleswig-holsteinischen Landtagswahl desselben Jahres war das Aufeinandertreffen eines Mannes (Volker Rühe) und einer Frau (Heide Simonis) als Spitzenkandidaten der Anlass zu einer empirischen Untersuchung. Der Erstautor der vorgenannten Studie hatte schon bei der nordrhein-westfälischen Landtagswahl 1995 die Informationssuche („Information seeking“) der Kölner Wähler untersucht ${ }^{16}$.

Der damit umrissene Forschungsstand zu Landtagswahlkämpfen ist bislang ziemlich unsystematisch und zumal hinsichtlich der Wahlkampfkommunikation über punktuelle Erkenntnisse nicht hinausgekommen. Dieser Umstand motiviert, die Berichterstattung der Tagespresse bei gleich zwei Landtagswahlen 2011 in den Blick zu nehmen und die Ergebnisse dieser Untersuchung mit jüngsten Wahlkampfstudien zur Bundestagswahl und zur EP-Wahl zu vergleichen. Erstmals sollten damit Wahlen auf drei Ebenen des politischen Systems miteinander verglichen werden, und zwar auf der regionalen (Landtagswahlen), der nationalen (Bundestagswahlen) und der supranationalen (EP-Wahlen) ${ }^{17}$.

\title{
1. Rangordnung der Wahlen und der Wahlkampfberichterstattung
}

Als die ersten Direktwahlen zum EP stattfanden, trafen Karlheinz Reif und Hermann Schmitt eine grundlegende Unterscheidung zwischen „first-order“ und „second-order elections“:

\begin{abstract}
„Second-order elections are, of course, neither new nor unfamiliar either in the European Community or in Western democracies as a whole. In each of these systems one can discern with relative ease which are the decisive elections: the 'first order' elections in parliamentary systems are the national parliamentary elections, and in presidential systems, the national presidential elections. In addition to these, however, there is a plethora of 'second order' elections: by-elections, municipal elections, various sorts of regional elections, those to a second chamber and the like." 18
\end{abstract}

14 Vgl. Ulrich Sarcinelli / Heribert Schatz (Hrsg.), Mediendemokratie im Medienland? Inszenierungen und Themensetzungsstrategien im Spannungsfeld von Menschen und Parteieliten am Beispiel der nordrhein-westfälischen Landtagswahl 2000, Opladen 2002.

15 Vgl. Frank Marcinkowski / Jörg-Uwe Nieland, Medialisierung im politischen Mehrebenensystem. Eine Spurensuche im nordrhein-westfälischen Landtagswahlkampf, in: Ulrich von Alemann I Stefan Marschall (Hrsg.), Parteien in der Mediengesellschaft, Wiesbaden 2002, S. 81 - 115; Markus Klein / Dieter Ohr / Stefanie Heinrich, Spitzenkandidaten im Wahlkampf. Die Veränderbarkeit von Kandidatenimages durch Wahlkampf und Medien, untersucht am Beispiel der nordrhein-westfälischen Landtagswahl am 14. Mai 2000, in: Publizistik, 47. Jg. (2002), S. 412 - 435.

$16 \mathrm{Vgl}$. Dieter Ohr / Peter R. Schrott, Campaigns and Information Seeking. Evidence from a German State Election, in: European Journal of Communication, 16. Jg. (2001), H. 4, S. $419-449$.

17 Zumindest ein Vergleich auf den beiden zuerst genannten Ebenen wurde bisher schon einmal durchgeführt, vgl. Jens Tenscher / Sonja Schmid, Berichterstattung nach Wahl. Eine vergleichende Analyse von Bundes- und Landtagswahlkämpfen in der Regionalpresse, in: Medien \& Kommunikationswissenschaft, 57. Jg. (2009), H. 1, S. $56-77$.

18 Karlheinz Reif/ Hermann Schmitt, Nine National Second-Order-Elections: A Conceptual Framework for the Analysis of European Election Results, in: European Journal of Political Research, 8. Jg. (1980), H. 1, S. 3 - 44, S. 8. 
Die Autoren machten diese Unterscheidung vor allem an der unterschiedlichen Beteiligung an nationalen („first-order“) und EP- („second-order“) Wahlen fest. Sie fanden ihre Begrifflichkeit auch bei späteren Wahlen bestätigt, die dann auch von anderen Autoren verwendet wurde. So entstand daraus ein gängiges Konzept ${ }^{19}$. Während Reif ${ }^{20} 1984$ EP-Wahlen als „zusätzliche nationale Nebenwahlen“ bezeichnete und sie darin „den Kommunalwahlen, Regional- oder Generalratswahlen, Provinzial- oder Landtagswahlen, den verschiedenen Arten von Nachwahlen usw. “21 gleichstellte, sprach er zugleich von der Gefahr, „daß die Direktwahl zum Europäischen Parlament [...] zu einer Wahl ,dritter Klasse` zu verkommen “22 drohe. 1997 vollzog er dann explizit den Schritt zu einer Dreistufung von Wahlen: „In the eyes of citizens, SOE [Second-Order Elections] would be less important than FOE [FirstOrder Elections], but are still 'real elections'. They 'produce' a (local, regional, European) government, especially a Head of Government [...] 'TOE' [Third-Order Elections] 'produce' an assembly but have no impact on the 'production' of the representative government." 23 Da aus EP-Wahlen keine oder allenfalls nur indirekt eine „Regierung “ hervorgeht, müssten diese streng genommen dem letztgenannten der drei Typen zugeordnet werden.

Die zitierten Unterscheidungen sind in der politikwissenschaftlichen Forschung entwickelt worden und dienen zur Analyse spezifisch politischer Merkmale von demokratischen Wahlen (vor allem der Wahlbeteiligung). Sie können aber auch herangezogen oder adaptiert werden, wenn man Wahlen kommunikationswissenschaftlich untersucht. Einschlägige Studien gibt es zu nationalen Wahlen seit den 1940er Jahren und zu den EP-Wahlen, seitdem solche überhaupt stattfinden (1979). Obwohl hinsichtlich der Wahlkampfberichterstattung sogleich Unterschiede zwischen nationalen und EP-Wahlen festgestellt wurden, ist erst vor kurzem das Konzept von Reif und Schmitt in die Unterscheidung von "first-rate coverage“ und „second-rate coverage“24 umgemünzt worden. Offen ist, ob sich auch hier eine Dreistufung feststellen lässt, wenn man die Berichterstattung über regionale Wahlen mit einbezieht und wie sich diese darin niederschlägt. Die aufgeworfene Frage interessiert aus mehreren Gründen: Besteht auch in der Medienberichterstattung eine Hierarchie der Wahlebenen, und wie wirkt sich diese gegebenenfalls in der Wahlkampfberichterstattung aus? Welche Ähnlichkeiten oder Unterschiede zeigen sich in der Wahlkampfdarstellung auf den verschiedenen Ebenen? Daraus wiederum lässt sich auf die (mediale) Wahrnehmung dieser Wahlen in der Bevölkerung schließen. Und hinsichtlich der Rangordnung ist zu fragen, durch welche

19 Vgl. Pippa Norris, Second-Order Elections Revisited, in: European Journal of Political Research, 31. Jg. (1997), H. 1, S. 109 - 114; Hermann Schmitt, The European Parliament Elections of June 2004. Still Second-Order?, in: West European Politics, 28. Jg. (2005), H. 3, S. 650 - 679; Jason R. Koepke / Nils Ringe, The Second-Order Election Model in an Enlarged Europe, in: European Union Politics, 7. Jg. (2006), H. 3, S. 321 - 346; Simon Hix / Michael Marsh, Punishment or Protest? Understanding European Parliament Elections, in: The Journal of Politics, 69. Jg. (2007), H. 2, S. 495 - 510; Claes H. de Vreese / Edmund Lauf/ Jochen Peter, a.a.O. (Fn. 3), S. 129 - 147; Jesper Strömbäck / Lars W. Nord, a.a.O. (Fn. 4), S. 137 - 152.

20 Vgl. Karlheinz Reif, Nationale Regierungsparteien verlieren die Wahl zum Europäischen Parlament 1984, in: ZParl, 15. Jg. (1984), H. 3, S. 341 - 352; ders., National Electoral Cycles and European Elections, in: Electoral Studies, 3. Jg. (1984), H. 3, S. $244-255$.

21 Ebenda, Nationale Regierungsparteien, S. 343.

22 Ebenda, S. 352.

23 Ders., European Elections as Member State Second-Order Elections Revisited, in: European Journal of Political Research, 31. Jg. (1997), H. 1/2, S. 115 - 124, S. 121.

24 Vgl. Claes H. de Vreese / Edmund Laufl Jochen Peter, a.a.O. (Fn. 3), S. 129 - 147. 
Merkmale gegebenenfalls die Berichterstattung von „second-order“ und „third-order elections" voneinander und wie sie von "first-order elections" abweichen.

\section{Umstände und Anlage der Untersuchung}

Zur Prüfung der Frage, wie sich die Wahlkampfberichterstattung auf drei Ebenen des politischen Systems in Deutschland unterscheidet, werden im Folgenden drei Studien zusammengeführt. Sie sind getrennt voneinander entstanden, haben aber so weit wie möglich dasselbe Design und Instrumentarium verwendet: Mit einer Inhaltsanalyse wurde jeweils die Berichterstattung von vier Zeitungen über die Wahlkämpfe zu regionalen, nationalen und EP-Wahlen untersucht.

\subsection{Regional: Landtagswahlen 2011}

Wegen des unterschiedlichen Wahlzyklus in den Bundesländern und des Aufwands, der dafür erforderlich gewesen wäre, konnten nicht alle 16 Landtagswahlen in die Untersuchung einbezogen werden. Zumindest zwei wurden für die Analyse ausgewählt, um die Basis des Vergleichs zu verbreitern. Die Chance dazu bot sich, weil am 27. März 2011 sowohl in Rheinland-Pfalz als auch in Baden-Württemberg Landtage gewählt wurden. In RheinlandPfalz regierten die Sozialdemokraten seit 2006 mit absoluter Mehrheit, zuvor schon seit 1991 in einer Koalition mit den Liberalen. In Baden-Württemberg stellten die Christdemokraten bereits seit 58 Jahren die Regierung, seit 1992 ebenfalls in einer Koalition mit der FDP.

In beiden Ländern war die Ausgangslage 2011 unterschiedlich. Absehbar war, dass die SPD die absolute Mehrheit in Rheinland-Pfalz verlieren würde, aber zusammen mit den Grünen würde weiter regieren können. So geschah es dann auch nach der Wahl ${ }^{25}$. Anders hingegen in Baden-Württemberg, wo die Grünen seit langem besonders stark sind. Hier drohte eine Abwahl der CDU/FDP-Koalition, wobei die thematische Konstellation des Wahltermins eine entscheidende Rolle spielte. Die bisherigen Oppositionsparteien, Grüne und SPD, erlangten tatsächlich eine knappe Mehrheit und konnten eine Regierung bilden. Die CDU musste erstmals in die parlamentarische Opposition gehen ${ }^{26}$.

Die Konstellation des Wahltermins am 27. März 2011 war nicht nur geprägt durch Vorgänge und Probleme, die für die beiden Länder selbst von Bedeutung waren. Sie wurde auch überschattet durch internationale und nationale Ereignisse. Geradezu weltweite Konsequenzen hatte das Erdbeben am 11. März 2011 vor der japanischen Küste, das die Katastrophe im Kernkraftwerk in Fukushima auslöste. Diese Ereignisse führten in Deutschland zum Wiederaufleben eines heftigen Streits um die Atomenergie, nachdem die Bundesregierung noch im Jahr zuvor die Laufzeiten für die Kernkraftwerke verlängert hatte. Unter dem Druck der Begebenheiten in Japan wurde dann in kürzester Zeit eine Kehrtwende vollzogen ${ }^{27}$.

25 Vgl. Heiko Gothe, a.a.O. (Fn. 9), S. $764-783$.

26 Vgl. Oscar W. Gabriel/ Bernhard Kornelius, a.a.O. (Fn. 9), S. $784-804$.

27 Vgl. ebenda; Heiko Gothe, a.a.O. (Fn. 9), S. 764 - 783. 
Um die Berichterstattung zu beiden Landtagswahlkämpfen empirisch zu untersuchen, wurden jeweils vier regionale Tageszeitungen aus beiden Bundesländern einer Inhaltsanalyse unterzogen, und zwar in den letzten vier Wochen vor dem Wahltermin. Dabei handelte es sich um folgende Blätter: in Rheinland-Pfalz die Allgemeine Zeitung (AZ, Mainz), die Rheinpfalz (RP, Ludwigshafen), die Rhein-Zeitung (RZ, Koblenz) und der Trierische Volksfreund (TV, Trier); in Baden-Württemberg der Mannheimer Morgen (MM, Mannheim), die Stuttgarter Nachrichten (StN, Stuttgart), die Badische Zeitung (BZ, Freiburg) und der Südkurier (SK, Konstanz).

\subsection{National: Bundestagswahl 2009}

Die letzte Bundestagswahl fand am 27. September 2009 statt. In den vier Jahren vorher hatte eine Große Koalition aus CDU/CSU und SPD regiert. Beide Parteien, die in der Regel in Opposition zueinander agieren, waren diese Koalition 2005 eingegangen, weil anders keine regierungsfähige Mehrheit zustande gekommen wäre. Weil die SPD viele Stimmen eingebüßt hatte, hatte Gerhard Schröder den Platz des Regierungschefs Angela Merkel überlassen müssen. 2009 verschoben sich die Mehrheitsverhältnisse erneut. Die Christdemokraten konnten jetzt eine Koalition mit den Liberalen eingehen; die SPD musste nach erheblichen Stimmenverlusten in die Opposition.

Die Untersuchung der Medienberichterstattung erstreckt sich auch hier auf die letzten vier Wochen vor dem Wahltermin. Einer Inhaltsanalyse unterzogen wurden in diesem Fall vier Tageszeitungen mit überregionaler Verbreitung: die Frankfurter Rundschau (FR, Frankfurt), die Süddeutsche Zeitung (SZ, München), die Frankfurter Allgemeine Zeitung (FAZ, Frankfurt) und die Welt (DW, Berlin).

Diese Zeitungen werden in der Medienforschung bevorzugt für wissenschaftliche Untersuchungen ausgewählt, weil sie das politische Meinungsspektrum zwischen links (FR), Mitte-links (SZ), Mitte-rechts (FAZ) und rechts (DW) abdecken. Dabei haben die Frankfurter Rundschau und die Süddeutsche Zeitung auch eine starke regionale und lokale Verortung, stärker zumindest als die Frankfurter Allgemeine Zeitung und Die Welt, die zwar ebenfalls Lokal-/Regionalteile haben, überwiegend aber national verbreitet werden.

Im Vordergrund des Bundestagswahlkampfs 2009 stand die Finanz- und Wirtschaftskrise, die im Jahr zuvor durch Bankenzusammenbrüche (zunächst in den USA) ausgelöst worden war und weltweit Konsequenzen nach sich zog. Innenpolitisch war die Frage beherrschend, ob die Große Koalition fortgesetzt oder durch eine kleine ersetzt werden würde.

\subsection{Supranational: EP-Wahl 2009}

Auf supranationaler Ebene wird alle fünf Jahre das Europäische Parlament gewählt. In Deutschland sind dabei 99 von insgesamt 736 Abgeordneten zu bestimmen. Ihre Wahl ist in einem eigenen Gesetz geregelt. Die nationalen Parteien stellen für diese Wahl Kandidatenlisten auf. Die Personen, die diese Listen anführen, können als Spitzenkandidaten gelten. Die letzte EP-Wahl fand am 7. Juni 2009 statt. Die Wahlbeteiligung lag bei 43,3 Prozent, 0,3 Prozentpunkte höher als 2005. 1979, bei der ersten EP-Wahl, hatte sie bei 65,7 Prozent gelegen, ein Wert, der später nie wieder erreicht wurde. 
Die Untersuchung der Berichterstattung erstreckte sich wie bei den anderen Wahlen auf die letzten vier Wochen vor dem Wahltermin, und zwar ebenfalls auf die vier oben genannten großen Tageszeitungen mit überregionaler beziehungsweise nationaler Verbreitung.

Frühere Untersuchungen haben nachgewiesen, dass auch Wahlkämpfe zum EP primär durch nationale und innenpolitische Themen bestimmt werden. Sie werden insbesondere von der jeweiligen Opposition dazu benutzt, mit der amtierenden Regierung abzurechnen und ihr einen „Denkzettel“ zu verpassen. Das trifft zumeist auch auf Deutschland $\mathrm{zu}^{28} .2009$ war die Möglichkeit dazu aber in geringerem Maße gegeben, weil die beiden großen Parteien, CDU/CSU und SPD, auf Bundesebene in einer Koalition zusammenarbeiteten.

\subsection{Methode der Untersuchung}

Wie der Überblick über die methodische Anlage zeigt, wurden der Vergleich und die Inhaltsanalysen zu den verschiedenen Zeitpunkten und Wahlen so weit wie möglich analog gestaltet. Bei den regionalen Wahlen lag es nahe, regionale/lokale Tageszeitungen zu untersuchen, weil diese Wahlen außerhalb des jeweiligen Landes (und in überregionalen Blättern) nur eine relativ begrenzte Aufmerksamkeit finden. Im Falle der regionalen und nationalen Wahlen wurde jeweils ein Sample gebildet, in das jeder zweite Artikel aufgenommen wurde. Um zu den absoluten Häufigkeiten zu gelangen, mussten die Werte danach verdoppelt werden. Bei der EP-Wahl wurden dagegen alle relevanten Artikel einbezogen, weil zu befürchten war, dass sonst die Basis für die Untersuchung zu schmal ausfallen würde. Ausgewählt wurde jeder Artikel, der einen der Spitzenkandidaten oder die Wahlkampagne als Thema hatte. Berücksichtigt wurden Beiträge und Bilder, die auf der Titelseite, im politischen Teil, auf Kommentar- oder Reportageseiten oder im Ressort Vermischtes veröffentlicht wurden. In den Regionalzeitungen wurden zusätzlich die Lokal- und Regionalseiten berücksichtigt. Nicht codiert wurden die Ressorts Wirtschaft, Sport, Feuilleton sowie Pressestimmen, Anzeigenteil und Leserbriefspalten.

Die Inhaltsanalyse erstreckte sich auf zwei Ebenen: die Beiträge als solche und die Bilder. Die Kategorien der Inhaltsanalyse wurden so weit wie möglich gleich gehalten, um tatsächlich einen direkten Vergleich zu ermöglichen. Neben formalen Kategorien (Umfang, Platzierung, Autoren/Quellen, Darstellungsformen) wurden inhaltliche erfasst. Im Vordergrund standen dabei Aspekte der Personalisierung und der Darstellung der jeweiligen Spitzenkandidaten. Das verwendete Codebuch war zunächst für eine Langzeitstudie zu den deutschen Bundestagswahlen entwickelt worden ${ }^{29}$ und inspiriert durch eine sehr bekannt gewordene amerikanische Studie ${ }^{30}$. Für die anderen Wahlen wurden die Kategorien adaptiert. Die Reliabilität der Codierung, gemessen nach dem Holsti-Koeffizienten, war sehr gut bis hinreichend (Werte zwischen 1 und 0,72$)^{31}$.

28 Vgl. Jürgen Wilke / Carsten Reinemann, a.a.O. (Fn. 4), S. 299 - 322.

29 Vgl. dies., Kanzlerkandidaten in der Wahlkampfberichterstattung. Eine vergleichende Studie zu den Bundestagswahlen 1949 - 1998, Köln / Weimar / Wien 2000.

30 Vgl. Thomas E. Patterson, Out of Order, New York 1993.

31 Die Verwendung des Holsti-Koeffizienten wird zwar in der Kommunikationswissenschaft zum Teil kontrovers diskutiert, doch in diesem Fall wurde sich bewusst für dieses Maß entschieden. Denn auch die zufallskorrigierte Reliabilität nach Krippendorff ist in gewisser Weise problematisch: Dieses Maß kann bei (dichotomen) Variablen mit schiefer Verteilung oder mangelnder Varianz bei 


\section{Befunde}

\subsection{Umfang der Berichterstattung}

Der Umfang der Berichterstattung ist ein grundlegender Indikator für die Bedeutung, die einer Wahl durch die Massenmedien zugemessen wird. Tabelle 1 zeigt, in wie vielen Artikeln die untersuchten deutschen Zeitungen jeweils die regionalen, die nationale und die supranationale Wahl im Zeitraum von vier Wochen behandelt haben.

\begin{tabular}{|c|c|c|c|c|}
\hline & \multicolumn{2}{|c|}{ Regional } & \multirow{2}{*}{$\begin{array}{c}\text { National } \\
\text { Bundestagswahl } \\
2009\end{array}$} & \multirow{2}{*}{$\begin{array}{c}\text { Supranationa } \\
\text { Europawahl } \\
2009\end{array}$} \\
\hline & $\begin{array}{c}\text { Landtagswahl } \\
\text { Rheinland-Pfalz } 2011\end{array}$ & $\begin{array}{c}\text { Landtagswahl } \\
\text { Baden-Württemberg } \\
2011 \\
\end{array}$ & & \\
\hline $\begin{array}{l}\text { Anzahl der } \\
\text { Artikel }\end{array}$ & 348 & 630 & 604 & 192 \\
\hline $\begin{array}{l}\text { Anzahl der } \\
\text { Anschläge }\end{array}$ & 962.312 & 1.553 .842 & 2.108 .004 & 749.187 \\
\hline
\end{tabular}

Auf den ersten Blick scheint sich die Annahme nicht zu bestätigen, dass Regionalwahlen „second-order elections“ sind, da die meisten Artikel zur Wahl in Baden-Württemberg 2011 veröffentlicht worden sind (630), mehr sogar als zur Bundestagswahl 2009 (604). Doch ist dies in doppelter Weise zu relativieren. Zum einen, weil der Bundestagswahlkampf 2009 langfristig gesehen eine nur sehr unterdurchschnittliche Beachtung in der Presse fand. Im Vergleich zu den vorangegangenen Wahlen 2002 und 2005 erschienen fast nur halb so viele Artikel ${ }^{32}$. Zum anderen bestätigt sich die angenommene Rangfolge der Wahlen durchaus, wenn man sich den tatsächlichen Umfang der Berichterstattung - gemessen in Zeilenanschlägen - ansieht. Dann ist die Dominanz der Bundestagswahl als „first-order election“ klar zu erkennen.

Ein deutlicher Unterschied bestand 2011 zwischen den beiden Bundesländern. Über die jeweiligen Wahlen haben die Zeitungen in Rheinland-Pfalz viel weniger berichtet als die in Baden-Württemberg. Das dürfte mit dem größeren Spannungsgehalt der letzteren zu erklären $\operatorname{sein}^{33}$. Außer der Frage der Atomenergie hatte der Streit um den Neubau des Haupt-

der Codierung einer Variablen schlecht angewendet werden (vgl. Jens Vogelgesang / Michael Scharkow, Reliabilitätstests in Inhaltsanalysen. Eine Analyse der Dokumentationspraxis in Publizistik und Medien \& Kommunikationswissenschaft, in: Publizistik, 57. Jg. (2012), H. 3, S. 333 - 345). Solche Variablen kommen in der vorliegenden Analyse aber sehr häufig vor. Um auch für diese Variablen Reliabilitätskoeffizienten berechnen und interpretieren zu können, wurde auf die einfache Prozentübereinstimmung nach Holsti zurückgegriffen. Alle Codierer nahmen an dem Reliabilitiätstest teil.

32 Vgl. Jürgen Wilke / Melanie Leidecker, Ein Wahlkampf, der keiner war? Die Presseberichterstattung zur Bundestagswahl 2009 im Langzeitvergleich, in: Christina Holtz-Bacha (Hrsg.), Die Massenmedien im Wahlkampf. Das Wahljahr 2009, Wiesbaden 2010, S. 339 - 372.

33 Vgl. Oscar W. Gabriel/ Bernhard Kornelius, a.a.O. (Fn. 9), S. $784-804$. 
bahnhofs in der Landeshauptstadt Stuttgart („Stuttgart 21“) das öffentliche Meinungsklima in Baden-Württemberg seit vielen Monaten eskalieren lassen. Zudem war der Wahlausgang lange Zeit ziemlich offen und ein Regierungswechsel wahrscheinlich, was in Rheinland-Pfalz nicht zu erwarten war. Insofern dürfte die hohe Zahl der Beiträge der Zeitungen in BadenWürttemberg dieser besonderen Situation geschuldet sein. Eindeutig an dritter Stelle („thirdorder election") steht die Berichterstattung über den EP-Wahlkampf. Allerdings sind die Artikel hierzu durchschnittlich die längsten (3.841 Zeilenanschläge pro Artikel), gefolgt von der Bundestagswahl (3.490 Zeilenanschläge). Häufigkeit und Länge der Beiträge stehen bei der EP-Wahl in einem umgekehrten Verhältnis zueinander, was vielleicht auch mit dem Ablauf des Wahlkampfs und seiner Intensität zusammenhängt. Die Artikel zu den Regionalwahlen sind im Durchschnitt etwas kürzer (Rheinland-Pfalz: 2.765; Baden-Württemberg: 2.466 Zeilenanschläge pro Artikel).

\subsection{Verlauf und Dynamik der Berichterstattung}

Beim Verlauf beziehungsweise bei der Dynamik der Berichterstattung geht es darum festzustellen, wie sich die Artikel der Tageszeitungen auf die letzten vier Wochen vor der Wahl verteilen. Die Auswertung dazu zeigt Abbildung 1.

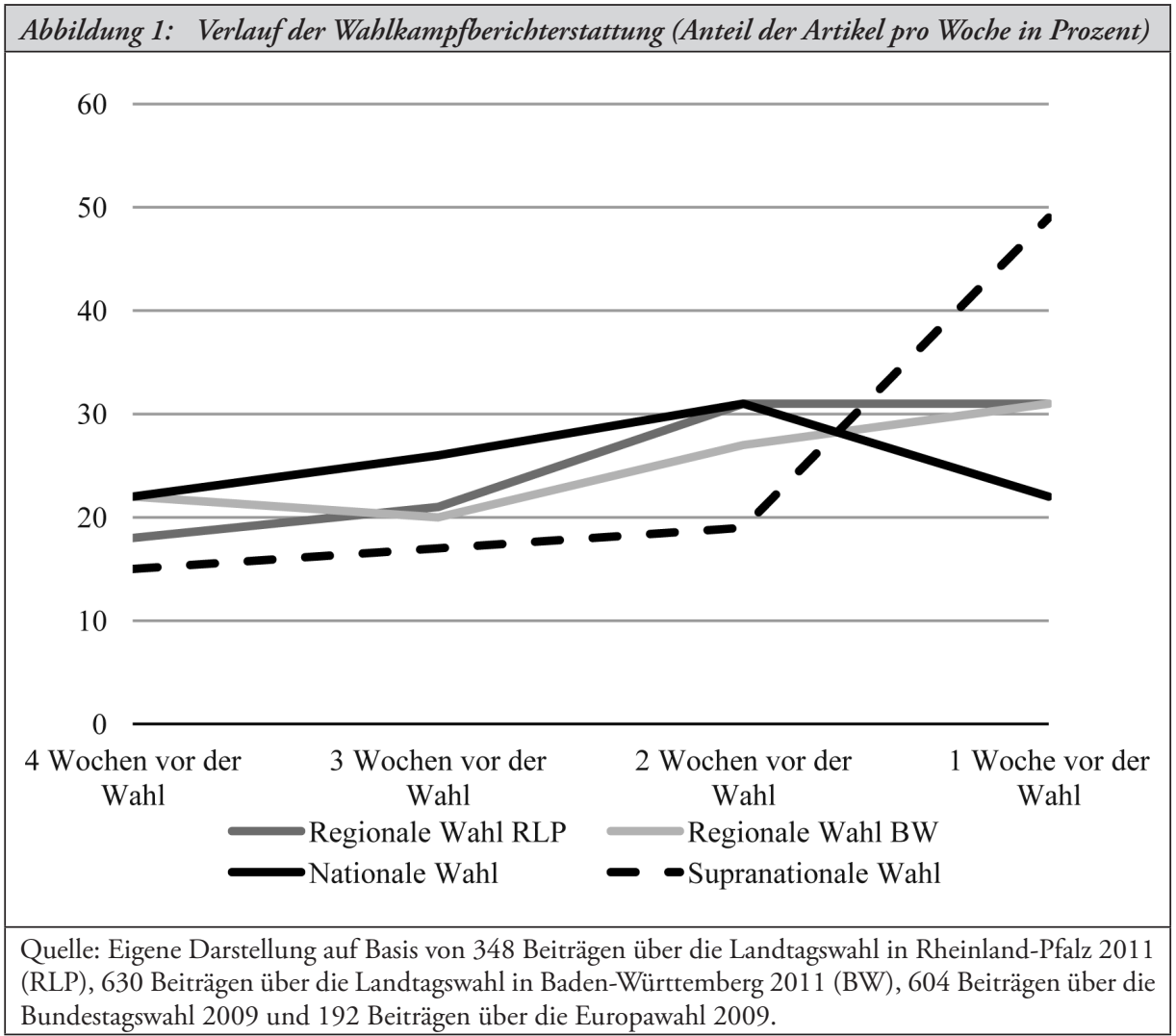


Der Verlauf der Berichterstattung in den vier Wochen der „heißen“ Phase des Wahlkampfs unterscheidet sich bei den drei Arten von Wahlen. Am meisten fällt auf, dass der EP-Wahlkampf in den ersten drei Wochen die geringste Aufmerksamkeit fand und dass diese erst in der Woche vor dem Wahltag anstieg. Fast die Hälfte aller Artikel erschien erst in der letzten Woche. Dagegen hatte die Berichterstattung über die Bundestagswahl ihren Höhepunkt schon in der dritten Woche. Ursache dafür war die Fernsehdebatte der Kanzlerkandidaten 14 Tage vor dem Wahltermin. In deren Folge wurden besonders viele Artikel veröffentlicht. Die auch in den Landtagswahlkämpfen veranstalteten Fernsehduelle der Spitzenkandidaten wurden in den Zeitungen viel weniger aufgegriffen. In der vierten Woche ging ihr Anteil schon wieder zurück. Die Dynamik der Berichterstattung über die Regionalwahlen liegt gewissermaßen dazwischen: Sie stieg vergleichsweise kontinuierlich bis zum Ende der Wahlkämpfe an und lag zuletzt auch in beiden Fällen dicht beieinander.

\subsection{Platzierung}

Auch die Platzierung der Wahlkampfartikel gibt Aufschluss über die Bedeutung, die die Journalisten den Wahlen zumessen (vgl. Abbildung 2). „First-order“-Wahlen sollten in den Zeitungen auffälliger platziert werden als „second-,, oder „third-order“-Wahlen.

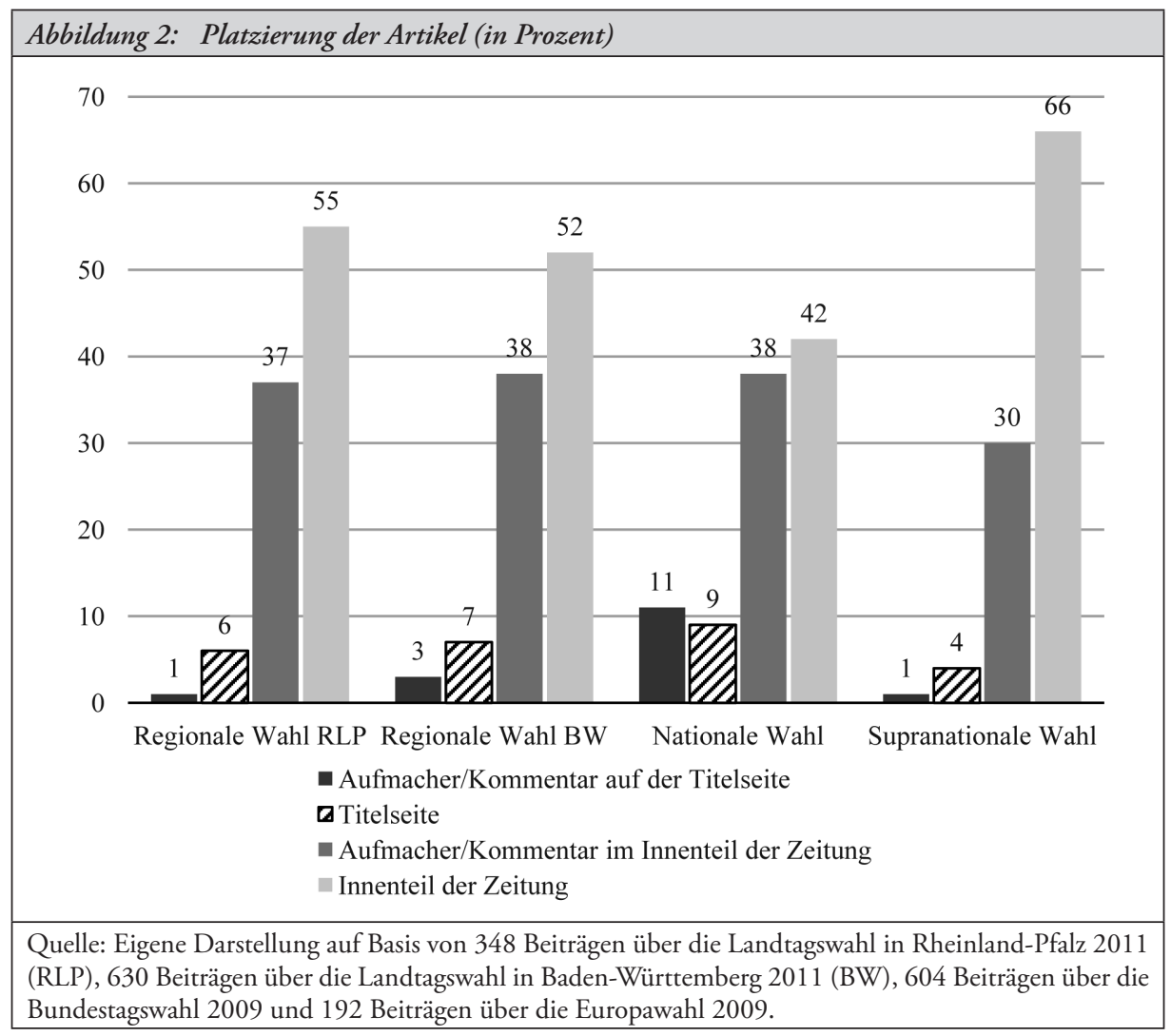


Die Befunde bestätigen tatsächlich die Dreistufung: Die unauffälligste Platzierung hatten die Artikel zur EP-Wahl. Zwei Drittel standen im Innenteil der Zeitungen, nur jeder dritte war ein Hauptartikel oder Kommentar im Innenteil. Am besten platziert wurden die Artikel zur Bundestagswahl: Jeder fünfte stand auf der ersten Zeitungsseite, jeder zehnte sogar als Aufmacher. Entsprechend geringer war der Anteil der Artikel im Inneren der Blätter. Die Regionalwahlen lagen wiederum zwischen der nationalen und der supranationalen Wahl. Sie schafften es seltener, zum Aufmacher zu werden oder auf Seite eins der Zeitungen zu erscheinen. Allerdings waren die Artikel zur Wahl in Baden-Württemberg wieder etwas besser platziert als diejenigen zur Wahl in Rheinland-Pfalz. Auch dies dürfte ein Reflex der unterschiedlichen politischen Bedeutung beider Wahlen gewesen sein.

\subsection{Darstellungsformen und Quellen}

Tageszeitungen behandeln Wahlkämpfe in verschiedenen journalistischen Formen und Formaten. Darüber, ob sie Unterschiede zwischen verschiedenen Arten von Wahlen machen, lässt sich vorweg schwerlich eine theoretisch begründete Hypothese aufstellen. Die Untersuchung zeigt gleichwohl, dass es solche Unterschiede gibt (vgl. Abbildung 3).

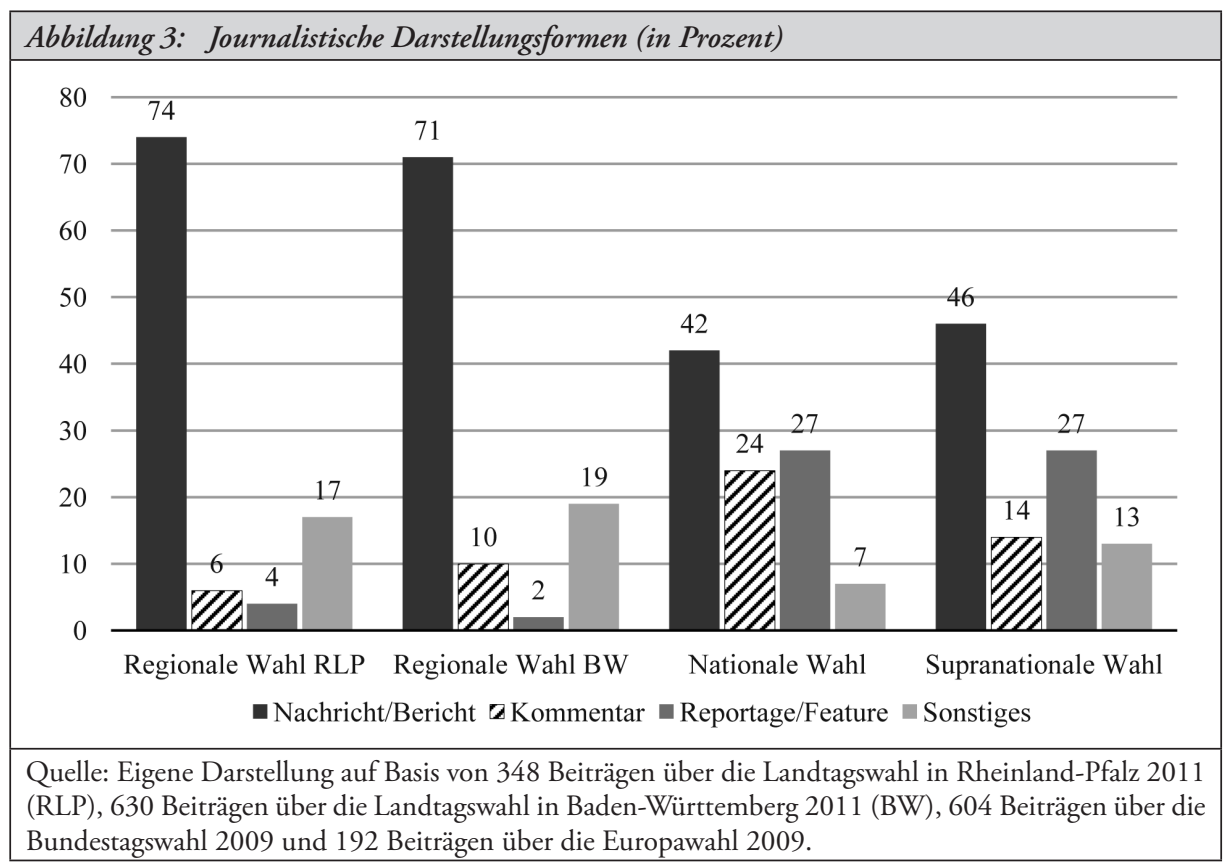

Die Grundform journalistischer Berichterstattung sind Nachrichten und Berichte, also Schilderungen, die den professionellen Regeln der Information folgen. Eindeutig dominiert diese Form in knapp drei Viertel der Fälle bei den Landtagswahlen. Bei der Bundestagswahl sind nur zwei Fünftel der Artikel von dieser Art, bei der EP-Wahl knapp die Hälfte. Dafür gibt es bei der nationalen Wahl einen viel höheren Anteil von Kommentaren. Auf suprana- 
tionaler Ebene haben diese wiederum einen „mittleren“ Anteil. Zwei Erklärungen sind möglich: Entweder steht bei Regionalwahlen tatsächlich die Information völlig im Vordergrund, während „subjektive“ Formen, insbesondere die Kommentierung, zurücktreten. Das spräche auch für einen geringeren Kontroversgehalt. Oder diese Merkmale sind generell für regionale Tageszeitungen charakteristisch, also durch den Zeitungstyp bedingt, der hier untersucht wurde. Bei Bundestagswahlen ist die Information nicht so dominant, sie werden in viel stärkerem Maße „subjektiv“ geschildert und kommentiert. Oder es handelt sich hier ebenfalls um generelle Merkmale der untersuchten Zeitungen mit überregionaler/nationaler Verbreitung. Bei den EP-Wahlen wurden dieselben Zeitungen untersucht. Hier ist der Anteil der „subjektiven“ Berichterstattung und Kommentierung geringer ausgeprägt als bei der nationalen Wahl. Es spricht also einiges dafür, dass es die spezifische Bedeutung der nationalen Parlamentswahl, also der „first-order election“, ist, die bedingt, dass sie journalistisch anders behandelt wird als „second-order“- und „third-order elections“. Die vorausgegangenen langfristigen Untersuchungen zu den deutschen Bundestagswahlen seit 1949 haben im Übrigen gezeigt, dass die „objektive“ Information durch Nachrichten und Berichte fast stetig zurückgegangen ist, während „subjektive“ Formen zugenommen haben ${ }^{34}$.

So gut wie keine Unterschiede gibt es hinsichtlich der Urheber der Wahlkampfartikel. Sie wurden bei allen drei Arten von Wahlen zu 76 bis 88 Prozent von den eigenen Journalisten der Tageszeitungen verfasst. Die politische Bedeutung der Ereignisse, um die es geht, machen diese zu einer ureigenen Sache der Redaktion. Fremdbeschafftes Material etwa durch Nachrichtenagenturen spielt nur eine nachrangige Rolle. Und dies gilt inzwischen selbst für nationale und supranationale Wahlen, wo es im Laufe der Jahrzehnte eine Steigerung der eigenständigen Berichterstattung gegeben hat ${ }^{35}$.

\subsection{Personalisierung}

Moderne Wahlkämpfe sind üblicherweise durch ein hohes Maß an Personalisierung gekennzeichnet. Unter Personalisierung versteht man, dass einzelne Akteure im Vordergrund der Medienberichterstattung stehen und verstärkt zum Deutungsmuster komplexer Sachverhalte sowie zum Anker von Bewertungen werden ${ }^{36}$. Insbesondere die Spitzenkandidaten, die sich im Fall der regionalen Wahlen für das Amt des Ministerpräsidenten, im Fall der nationalen Wahlen für das Amt des Bundeskanzlers und im Fall der supranationalen Wahlen als Spitzenkandidaten der Parteien für das EP bewerben, stehen dabei im Fokus der Medienberichterstattung. Da die EP-Kandidaten - im Gegensatz zu den Kanzlerkandidaten auf nationaler und den Ministerpräsidentenkandidaten auf regionaler Ebene - nicht für eine bestimmte Funktion oder ein Amt gewählt werden, kandidieren hier üblicherweise eher

34 Vgl. Jürgen Wilke / Carsten Reinemann, a.a.O. (Fn. 29); Jürgen Wilke / Melanie Leidecker, a.a.O. (Fn. 32), S. $339-372$.

35 Vgl. Jürgen Wilke / Carsten Reinemann, Auch in der Presse eine Nebenwahl? Die Berichterstattung über die Europawahlen 1979 - 2004 und die Bundestagswahlen 1980 - 2002 im Vergleich, in: Christina Holtz-Bacha (Hrsg.), Europawahl 2004. Die Massenmedien im Europawahlkampf. Wiesbaden 2005, S. 153 - 173, S. 165.

36 Vgl. Christina Holtz-Bacha, Bundestagswahlkampf 2002: Ich oder der?, in: dies. (Hrsg.), Die Massenmedien im Wahlkampf. Die Bundestagswahl 2002, Wiesbaden 2004, S. 9 - 28, S. 20. 
weniger prominente, jedoch verdiente Parteimitglieder „aus der zweiten Reihe“ 37 . Im Gegensatz dazu werden bei den Bundestags- oder Landtagswahlen - aufgrund der Bedeutung des zu bekleidenden Amtes - in der Regel Personen mit einem hohen Bekanntheitsgrad aufgestellt ${ }^{38}$. Daher ist zu schlussfolgern, dass die Berichterstattung über Wahlen auf regionaler und nationaler Ebene stärker personalisiert ist, als diejenige über Wahlen auf supranationaler Ebene. Zwischen der nationalen und der regionalen Wahlkampfberichterstattung ist eine weitere Abstufung hinsichtlich des Grades der Personalisierung zu erwarten, da die Reichweite der Regierungsmacht im Falle des Bundeskanzlers die gesamte Nation, im Falle des Ministerpräsidenten ein Bundesland betrifft.

Der Grad der Personalisierung der Wahlkampfberichterstattung wurde anhand der Menge der Beiträge, die einen Bezug zu mindestens einem Spitzenkandidaten enthalten, ermittelt. Obwohl dies eines der Kriterien war, anhand dessen die Beiträge der Tageszeitungen für die Inhaltsanalyse ausgewählt wurden, zeigen sich in der Presseberichterstattung unterschiedlich starke Bezüge zu den Kandidaten (vgl. Abbildung 4).

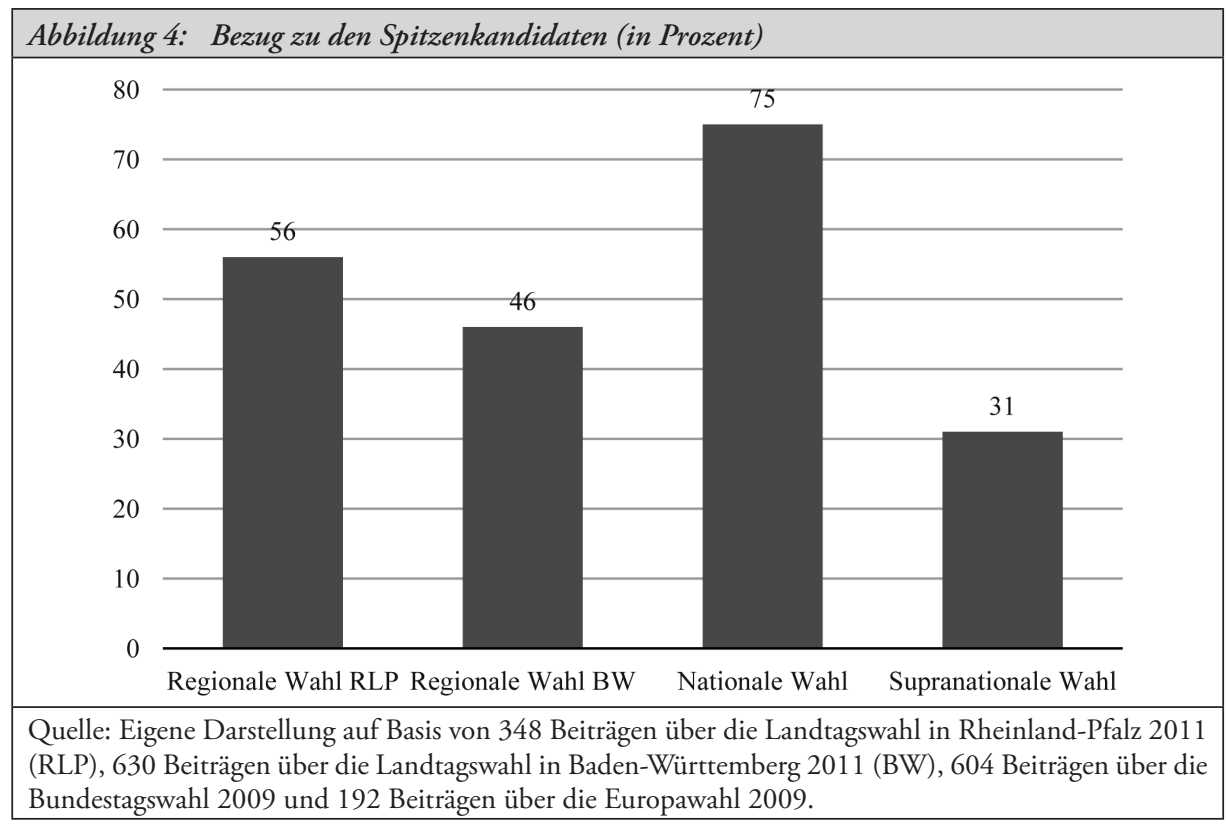

In der Tat erweist sich die Presseberichterstattung zur EP-Wahl als weniger personalisiert oder kandidatenfokussiert als diejenige zur Bundestagswahl oder zu den Landtagswahlen. Während bei der EP-Wahl knapp ein Drittel der Beiträge einen Bezug zu Spitzenkandidaten aufweist, nimmt die Berichterstattung zur Bundestagswahl mit drei Vierteln der Artikel diesbezüglich die Spitzenposition ein. Dazwischen liegen die Anteile der Beiträge mit Kandidatenbezug im Falle der regionalen Parlamentswahlen (Rheinland-Pfalz: 56 Prozent, Ba-

37 Peter Fahrenholz, Die Wahl im Schatten. Europawahl 2009, in: sueddeutsche.de vom 10. Mai 2010, http://www.sueddeutsche.de/politik/europawahl-die-wahl-im-schatten-1.448109 (Abruf am 12. März 2013).

38 Vgl. Jürgen Wilke / Christian Schäfer / Melanie Leidecker, a.a.O. (Fn. 4), S. 155 - 179. 
den-Württemberg: 46 Prozent). Dass der Anteil der Artikel, die einen Kandidatenbezug enthalten, bei beiden Landtagswahlen unterschiedlich groß ist, dürfte darauf zurückzuführen sein, dass der Wahlkampf in Baden-Württemberg mehr von vielen strittigen Themen (beispielsweise in der Verkehrs- und Umweltpolitik) beherrscht war. Die Personalisierung war deshalb dort weniger stark ausgeprägt und - weil es im Grunde drei Spitzenkandidaten gab - weniger antagonistisch oder bipolar fokussiert.

\subsection{Bebilderung}

Als weiteren Indikator für eine personalisierte Wahlkampfberichterstattung lässt sich heranziehen, wie viele Bilder der Spitzenkandidaten die Zeitungen in den vier Wochen vor den Wahlen veröffentlichten. Bereits mehrfach wurde in den letzten Jahren ein Trend zu einer stärker visualisierten Wahlkampfberichterstattung sowohl im Fernsehen ${ }^{39}$ als auch in der gedruckten Tagespresse ${ }^{40}$ festgestellt ${ }^{41}$. Diese zunehmende Visualisierung vermittelt den Wählern einen lebendigeren und stärker personalisierten Eindruck vom Wahlkampf ${ }^{42}$. Entsprechend der weiter oben erwähnten größeren Bedeutung, die den Spitzenkandidaten bei nationalen und regionalen Wahlen im Vergleich zur EP-Wahl zukommt, war anzunehmen, dass diese auch innerhalb der Presseberichterstattung zur nationalen Wahl und zu den regionalen Wahlen häufiger im Bild zu sehen sind als in den Beiträgen zur supranationalen EP-Wahl.

Abbildung 5 erweckt auf den ersten Blick den Eindruck, dass sich die Unterschiede in der Intensität der Wahlkampfberichterstattung auch in der Visualisierung zeigen. Die meisten Fotos wurden zur Bundestagswahl, die wenigsten zur EP-Wahl veröffentlicht. Wiederum liegen die Werte für die regionalen Wahlen dazwischen. Dieser Eindruck ist aber zu korrigieren, wenn man die verschieden umfangreiche Berichterstattung in Betracht zieht und die Anzahl der Bilder pro Beitrag berechnet. Dann erscheint die Berichterstattung über den regionalen Wahlkampf in Rheinland-Pfalz visuell am stärksten personalisiert. Etwa jeder vierte Beitrag über die Wahlen in den Bundesländern enthielt ein Bild, auf dem mindestens einer der Spitzenkandidaten zu sehen ist (Rheinland-Pfalz: 0,25 Bilder pro Beitrag). Im Vergleich dazu enthielt nur etwa jeder achte Beitrag in den vier Wochen vor der baden-württembergischen Wahl Bilder der Kandidaten (Baden-Württemberg: 0,13 Bilder pro Beitrag). Die dortigen Zeitungen veröffentlichten damit sogar weniger Bilder als die überregionalen Zeitungen in den vier Wochen vor der EP-Wahl: Sie publizierten im Durchschnitt 0,18 Bilder der EP-Kandidaten pro Beitrag (was bedeutet, dass jeder fünfte Beitrag ein Bild eines Kandidaten enthielt). Dass zur Landtagswahl in Rheinland-Pfalz die meisten Fotos veröffentlicht wurden, hat möglicherweise mit dem Typ der regionalen Abonnementzeitung zu tun, mit der größeren „Nähe“ des Wahlkampfs und seiner Kandidaten sowie dem relativ hohen Anteil der Beiträge, die einen Kandidatenbezug enthalten. Die Tatsache, dass die Beiträge zur regionalen

39 Vgl. Winfried Schulz / Reimar Zeh, Die Kampagne im Fernsehen - Agens und Indikator des Wandels. Ein Vergleich der Kandidatendarstellung, in: Christina Holtz-Bacha (Hrsg.), Die Massenmedien im Wahlkampf. Die Bundestagswahl 2005, Wiesbaden 2006, S. 277 - 305.

40 Vgl. Jürgen Wilke, Die Visualisierung der Wahlkampfberichterstattung in Tageszeitungen 1949 bis 2009, in: ders., Von der frühen Zeitung zur Medialisierung. Gesammelte Studien II, Bremen 2001, S. $183-211$.

41 Marcus Maurer / Carsten Reinemann, Medieninhalte. Eine Einführung, Wiesbaden 2006, S. 128.

42 Vgl. Winfried Schulz / Reimar Zeh, a.a.O. (Fn. 39), S. 288. 


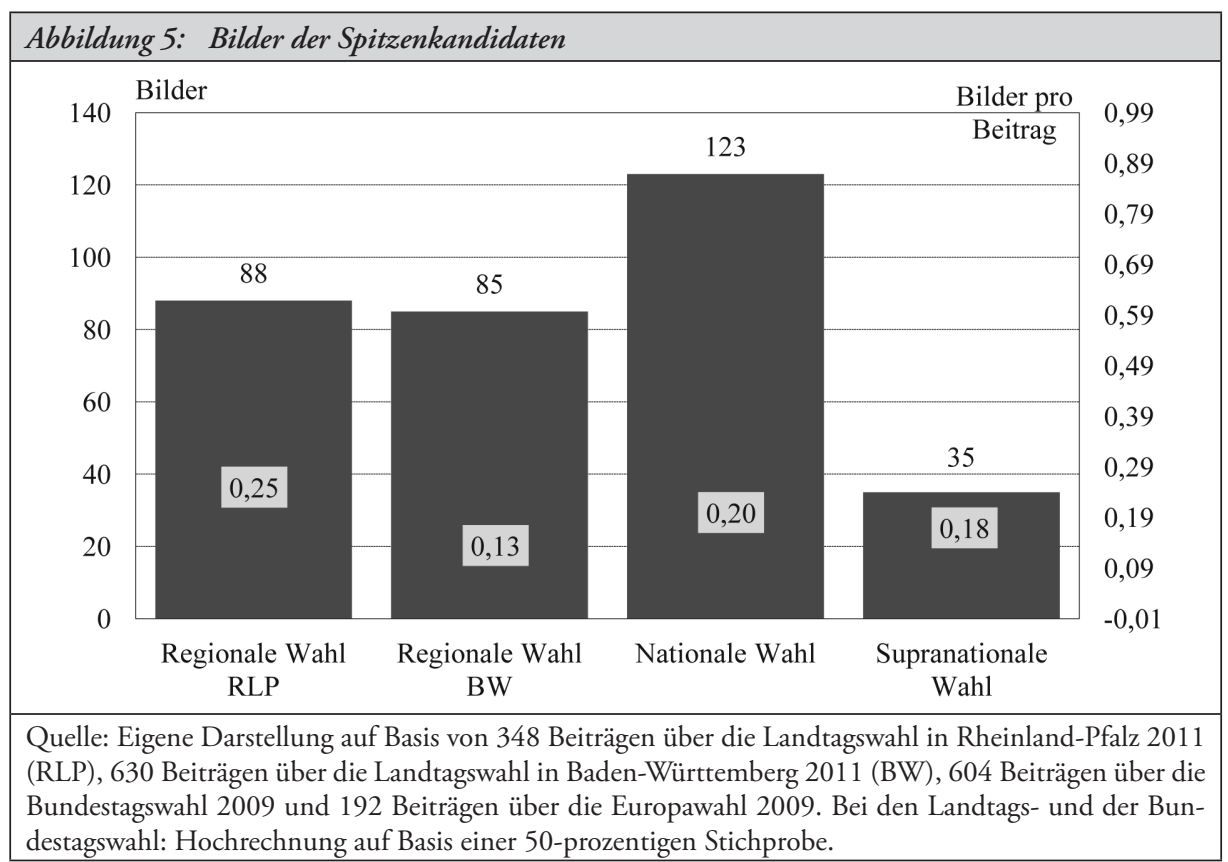

Wahl in Baden-Württemberg weniger illustriert waren, kann durch die große Menge kurzer Artikel erklärt werden, die üblicherweise keinen Raum für Bilder lassen.

\subsection{Authentizität}

Je häufiger die journalistischen Beiträge einen Bezug zu den Kandidaten enthalten, die zur Wahl stehen, desto wahrscheinlicher ist auch, dass Aussagen der Kandidaten in den Artikeln wörtlich wiedergegeben werden. Gleichzeitig erscheint die Presseberichterstattung umso authentischer, je mehr sie Botschaften der Kandidaten in deren eigenen Worten - sozusagen im O-Ton - wiedergibt anstelle mit den Worten eines Journalisten. Weil die Berichterstattung über die nationale Parlamentswahl den höchsten Kandidatenbezug besitzt, ist auch zu erwarten, dass bei ihr die Kandidaten am meisten zitiert werden. Landtagswahlen sollten, entsprechend ihres Kandidatenbezugs, auch hier eine mittlere Position einnehmen.

Tatsächlich weist die Berichterstattung über die Bundestagswahl mit 28 Prozent den höchsten Anteil an Beiträgen mit Zitaten der Spitzenkandidaten auf, diejenige über die EPWahl hingegen den geringsten (mit 18 Prozent der Beiträge). Die Berichterstattung über die regionalen Wahlen liegt mit Werten von 21 (Baden-Württemberg) und 24 Prozent (Rheinland-Pfalz) zwischen der nationalen und der supranationalen.

Der Umfang der Kandidatenzitate (gemessen in Zeilen) bestätigt diese Rangfolge (vgl. Abbildung 6). In der Bundestagswahlberichterstattung wurden die Kandidaten mit insgesamt 3.222 Zeilen zitiert. Rang zwei belegen die Beiträge zu den Regionalwahlen: In Rheinland-Pfalz enthalten sie insgesamt 2.212 Zeilen und in Baden-Württemberg 2.000 Zeilen mit Zitaten der Kandidaten. Am wenigsten werden - mit lediglich 849 Zeilen - die EP-Kandidaten zitiert. 


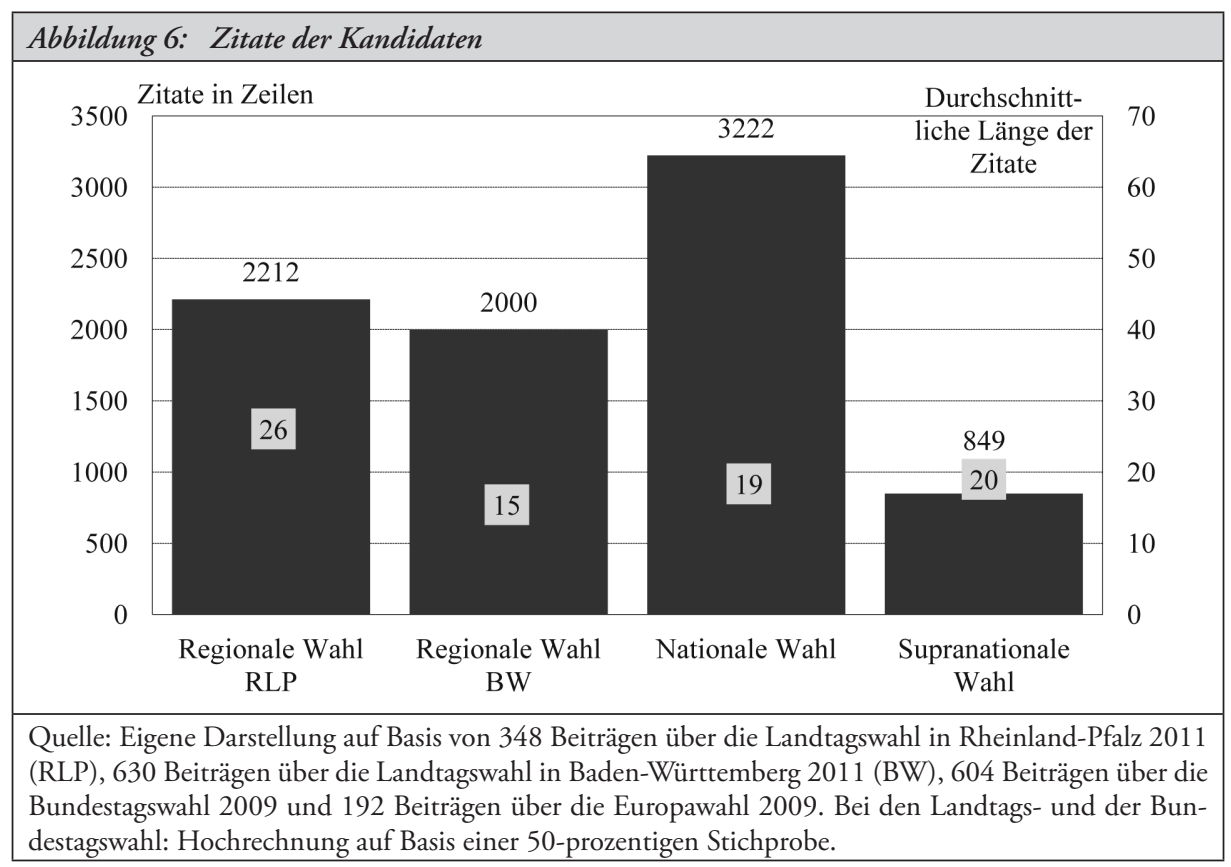

Da zu den vier genannten Wahlen unterschiedlich viele Beiträge veröffentlicht wurden, sind die oben genannten Befunde allein nicht aussagekräftig genug. Berechnet man die durchschnittliche Länge der Kandidatenzitate (indem man jeweils die Gesamtlänge der Zitate durch die Anzahl der Beiträge dividiert, in denen sie vorkommen), so wird deutlich, dass die EP-Kandidaten nicht am kürzesten zitiert werden, sondern dass die durchschnittliche Länge der Zitierung mit derjenigen von Bundestagskandidaten vergleichbar ist. Am geringsten fällt der Umfang der Kandidatenzitate bei der Landtagswahl in Baden-Württemberg aus (mit einer durchschnittlichen Länge von nur 15 Zeilen). Auffällig ist ihre Länge in der Presseberichterstattung zur regionalen Wahl in Rheinland-Pfalz: Hier werden die Kandidaten mit durchschnittlich 26 Zeilen am ausführlichsten zitiert. Kurt Beck und Julia Klöckner hatten damit die größte Chance, den Lesern ihre Standpunkte in eigenen Worten zu vermitteln. In dieser Hinsicht weist die Berichterstattung zur Landtagswahl in Rheinland-Pfalz den höchsten Grad an „Authentizität“ auf. Wie ist dies zu erklären? Abbildung 3 zeigt deutlich, dass der Anteil „sonstiger“ journalistischer Darstellungsformen im Falle der beiden Landtagswahlen relativ hoch war (Rheinland-Pfalz: 17 Prozent; Baden-Württemberg: 19 Prozent). Bei der Wahlberichterstattung in Rheinland-Pfalz ist dieser Wert unter anderem auf relativ viele Porträts (acht Prozent) und Interviews (fünf Prozent) der Kandidaten zurückzuführen. Gerade diese journalistischen Formen enthalten direkte Aussagen der Kandidaten.

\subsection{Bewertung der Kandidaten}

Zuletzt interessiert, welches Bild von den Spitzenkandidaten in der Wahlkampfberichterstattung vermittelt und wie diese in den verschiedenen Wahlkämpfen bewertet wurden (vgl. 

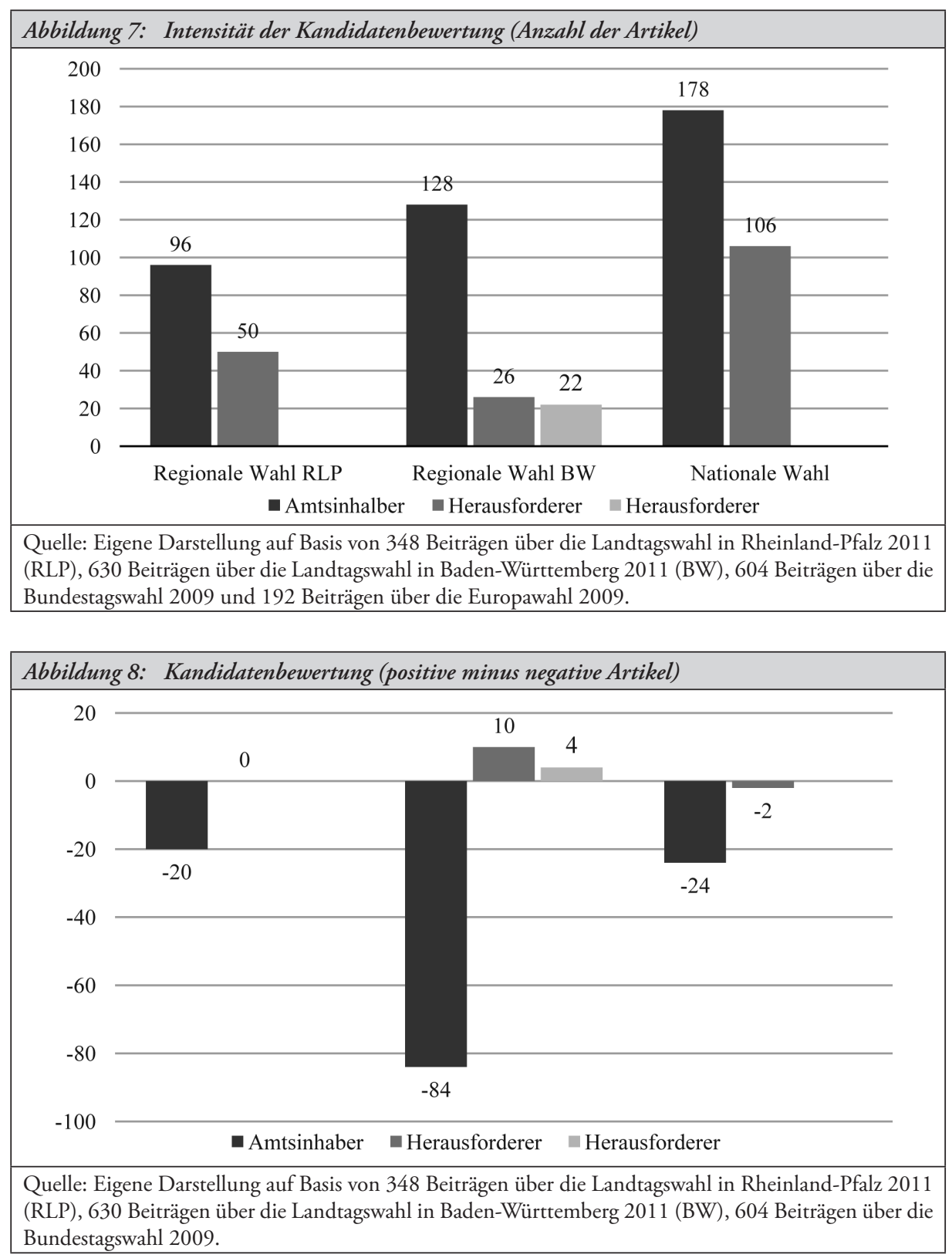

Abbildungen 7 und 8), und zwar nur auf regionaler und Bundesebene, da bei der EP-Wahl keine Bewertungen der Kandidaten (wegen deren geringer Präsenz) erfasst wurden.

Gemäß obiger Definition enthielt ein Artikel eine Bewertung, wenn zumindest einem Kandidaten eine positive oder negative Eigenschaft zu- oder abgesprochen wurde. Die Balken in Abbildung 7 zeigen die Zahl der Artikel, die Bewertungen der Kandidaten enthielten (unterteilt in Amtsinhaber und Herausforderer). Die Landtagswahl in Baden-Württemberg 
ist insofern ein Sonderfall, als hier - im Gegensatz zum rheinland-pfälzischen Fall und zur Bundestagswahl - gleich zwei Herausforderer (Wilfried Kretschmann und Nils Schmid) eine reelle Chance auf das Amt des Ministerpräsidenten besaßen.

Gemeinsam ist allen drei Wahlkämpfen, ob regional oder national, ein Amtsinhaberbonus in der Berichterstattung ${ }^{43}$. Die Zeitungsbeiträge bewerten den amtierenden Kanzler/ Ministerpräsidenten immer häufiger als seine(n) Herausforderer, und zwar unabhängig von der Rangordnung der Wahlen.

Bei der Differenzierung in positive und negative Artikel ist zu erkennen, dass die Amtsinhaber (Bundeskanzler, Ministerpräsidenten) häufiger bewertet wurden, zugleich aber auch negativer als ihre Herausforderer. Dieser Befund zeigt sich unabhängig von der Hierarchie der Wahlebenen. Die Parteizugehörigkeit der Kandidaten spielte keine Rolle für die Bewertung. Besonders negativ wurde 2011 der Amtsinhaber bei der Landtagswahl in Baden-Württemberg, Stefan Mappus, beurteilt. Er wurde bereits seit seinem Amtsantritt im Februar 2010 aufgrund seiner „polarisierenden Art“ immer wieder kontrovers in der Öffentlichkeit ${ }^{44}$ diskutiert. Im Vergleich dazu kamen seine beiden Herausforderer deutlich positiver im Urteil der Presse weg.

\section{Zusammenfassung: Drei Ebenen der Wahlkampfberichterstattung}

Wie eingangs dargelegt, wurde die in der Politikwissenschaft etablierte Typologie von „first order“- und „second order“-Wahlen ${ }^{45}$ später in der kommunikationswissenschaftlichen Forschung aufgriffen ${ }^{46}$. Aufgrund der von ihnen konstatierten Unterschiede in der Berichterstattung über nationale und EP-Wahlen unterschieden Claes de Vreese, Edmund Lauf und Jochen Peter (in Anlehnung an Karlheinz Reif und Hermann Schmitt) zwischen einer „firstrate coverage“ (über nationale Wahlen) und einer „second-rate coverage“ (über EP-Wahlen). Bezieht man jedoch zusätzlich die Berichterstattung über die in Deutschland stattfindenden Landtagswahlen ein, muss die Differenzierung von „first“- und „second-rate coverage“ ergänzt beziehungsweise erweitert werden. Die hier vorgelegten Befunde zeigen, dass die Berichterstattung zur EP-Wahl und zu Landtagswahlen nicht auf derselben Ebene verortet werden können. Vielmehr liegen regionale Wahlen bei den meisten herangezogenen Indikatoren der Berichterstattung zwischen der nationalen und supranationalen Wahl.

Über regionale Wahlen wird (1) umfangreicher berichtet als über die EP-Wahl, jedoch weniger umfangreich als über die Bundestagswahl. Über die beiden regionalen Wahlen wurde (2) kontinuierlicher berichtet als über die EP-Wahl, und die Beiträge über die regionalen Wahlen in der regionalen Presse wurden (3) prominenter platziert als diejenigen über die EP-Wahl in der überregionalen Presse. Ferner wurden die europäischen Spitzenkandidaten seltener in den Beiträgen erwähnt (4) und zitiert (5); sie waren auch seltener im Bild zu sehen als diejenigen der regionalen Wahlen (6). Am häufigsten wurden - absolut gesehen - die Spitzenkandidaten der Bundestagswahl in der Presseberichterstattung erwähnt, abgebildet und zitiert (7).

43 Vgl. Jürgen Wilke / Carsten Reinemann, a.a.O. (Fn. 29).

44 Vgl. Oscar W. Gabriel/ Bernhard Kornelius, a.a.O. (Fn. 9), S. $784-804$.

45 Vgl. Karlheinz Reifl Hermann Schmitt, a.a.O. (Fn. 18), S. 3 - 44.

46 Vgl. Claes H. de Vreese / Edmund Lauf/ Jochen Peter, a.a.O. (Fn. 3), S. 129 - 147 
Es mag sich die Frage aufdrängen, ob die aufgetretenen Unterschiede (mit) darauf zurückzuführen sind, dass regionale mit überregional verbreiteten Zeitungen verglichen wurden, sie also dem Zeitungstyp geschuldet sind. Denn allein der Umfang der politischen Berichterstattung ist bei den überregionalen Blättern meist generell größer. Dennoch wäre das Heranziehen überregionaler Zeitungen zur Analyse der Landtagswahlberichterstattung (um den Zeitungstyp gleich zu halten) keine geeignete Alternative gewesen, da überregionale Blätter wenig über Landtagswahlkämpfe berichten und sogar viel weniger als die jeweiligen Tageszeitungen des betreffenden Bundeslandes. Umgekehrt ist auch nicht zu erwarten, dass die Regionalpresse die Bundestagswahlen, die über die Machtverteilung auf Bundesebene entscheiden, weniger behandelt und die Landtagswahlkämpfe mehr. Das hat schon die Studie von Jens Tenscher und Sonja Schmid belegt, der zufolge 2005/2006 sechs Regionalzeitungen aus drei Bundesländern durchschnittlich halb so viele Beiträge den Landtagswahlen wie den Bundestagswahlen widmeten ${ }^{47}$. Somit dürfte vermutlich auch eine Analyse regionaler Zeitungen hinsichtlich der Bundestagswahlberichterstattung zu keinem anderen Ergebnis führen, als dass über Landtagswahlen weniger berichtet wird als über Bundestagswahlen.

Auf der Basis der Befunde erscheint es angebracht, für die Wahlkampfberichterstattung drei Ebenen zu unterscheiden:

- erstrangige Berichterstattung ("first-rate coverage") bei Bundestagswahlen,

- zweitrangige Berichterstattung („second-rate coverage”) bei Landtagswahlen und

- drittrangige Berichterstattung („third-rate coverage”) bei EP-Wahlen.

Die drei Ebenen des politischen Systems, auf denen in Deutschland Wahlen stattfinden (regional, national, supranational), bestimmen demzufolge maßgeblich die Medienberichterstattung über die entsprechenden Wahlkämpfe. Das mag nicht überraschen und eine „gefühlte" (und durch die übliche Wahlbeteiligung manifestierte) Differenz bestätigen. Aber vielleicht ist das Ausmaß der Berichterstattung eine der Ursachen für das Interesse an den Wahlen und für die Kluft zwischen den Wahlbeteiligungsraten.

Im Übrigen zeigten sich auch einige Merkmale der Berichterstattung, die unabhängig sind von der Art der Wahl und nicht durch die Ebenen des politischen Systems determiniert zu sein scheinen. So unterschieden sich die Wahlen nicht (oder nur wenig) bei den journalistischen Darstellungsformen und bei den Urhebern der Beiträge. Ferner wurden sowohl bei der nationalen Wahl als auch bei den regionalen Wahlen die Amtsinhaber (Kanzler, Ministerpräsidenten) immer häufiger, zugleich aber auch negativer bewertet als ihr(e) Herausforderer. Diese Aspekte scheinen sozusagen Konstanten der Wahlkampfberichterstattung zu sein. Solche Konstanten werden schließlich noch durch aktuelle Umstände überlagert: Das zeigte sich 2011 beispielsweise in der durch „Stuttgart 21" und die Kernenergie-Debatte bedingten, ganz besonders negativen Bewertung des Spitzenkandidaten der CDU im Wahlkampf in Baden-Württemberg ${ }^{48}$. 